\title{
Editorial
}

\section{La humanización del cuidado a través de las narrativas y la poesía como producto de la investigación aplicada}

The humanization of care through narratives and poetry as a product of applied research A humanização do cuidado através de narrativas e poesia como produto da pesquisa aplicada

\author{
José Siles González
}

Catedrático de la Facultad de Ciencias de la Salud. Universidad de Alicante

Cómo citar este artículo en edición digital: Siles-González, J. (2018). La humanización del cuidado a través de las narrativas y la poesía como producto de la investigación aplicada. Cultura de los Cuidados (Edición digital), 22(52). Recuperado de http://dx.doi.org/10.14198/cuid.2018.52.01

Correspondencia: Departamento de enfermería. Universidad de Alicante. Apartado Correos: 03080. Alicante (España)

Correo electrónico: jose.siles@ua.es

Recibido: Editorial invitada; Aceptado: Editorial invitada.

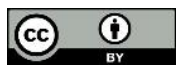

\section{ABSTRACT}

This editorial aims to clarify the importance of the humanization of nursing care as a product of applied research. There has been a reflection on the need for a change of scientific paradigm to understand the fact that the humanization of care implies an optimization of nursing practice and the need to use methodologies such as narratives and poetry in the nursing context.

Keywords: Humanization of care, narratives and nursing, care poetry, sociocritical paradigm.

\section{RESUMO}

Este editorial visa esclarecer a importância da humanização do cuidado de enfermagem como produto da pesquisa aplicada. Houve uma reflexão sobre a necessidade de uma mudança de paradigma científico para compreender o fato de que a humanização do cuidado implica uma otimização da prática de enfermagem e a necessidade de utilizar metodologias como narrativas e poesias no contexto da enfermagem.

Palavras-chave: Humanização do cuidado, narrativas e enfermagem, poesia assistencial, paradigma sociocrítico.

\section{RESUMEN}

Esta editorial tiene el objetivo de clarificar la importancia de la humanización de los 
cuidados en enfermería como producto de la investigación aplicada. Se ha realizado una reflexión sobre la necesidad de un cambio de paradigma científico para entender el hecho de que la humanización de los cuidados implica una optimización de la práctica de enfermería y la necesidad de utilizar metodologías como las narrativas y la poesía en el contexto enfermero.

Palabras clave: Humanización de los cuidados, narrativas y enfermería, poesía de los cuidados, paradigma sociocrítico.

\section{Clarificando cuestiones previas}

El impulso que ha motivado esta editorial está enraizado en dos circunstancias que se mancomunan de forma tangible en el contexto de la última reunión organizada por la Fundación Índex este último mes de noviembre en Granada: Por un lado, el profundo interés en la temática de la humanización de los cuidados desarrollado durante décadas y las metodologías pertinentes para potenciarla (investigación cualitativa, narrativas, etcétera); por otro, y tal vez sea el auténtico desencadenante, la dificultad expresada por una enfermera para entender con cierta precisión lo que significa la humanización de los cuidados para la disciplina tanto a nivel profesional como científico. Ante la afirmación de que la humanización de los cuidados debería constituirse en un producto esencial de la investigación aplicada y/o traslacional en enfermería, la alumna en cuestión opinaba que la auténtica investigación aplicada debería producir resultados para mejorar la práctica profesional; es decir, no entendía que la humanización de los cuidados constituyera un producto que mejoraba la práctica profesional. Sin duda esta dificultad para entender esta cuestión se debe a una ubicación paradigmática diferente cuya perspectiva no contempla el sinónimo entre humanización del cuidado y optimización de la práctica profesional. En el paradigma neopositivista (racional tecnológico), el producto de la investigación aplicada se ciñe estrictamente a la tecnología y a las necesidades derivadas de su aplicación por parte del personal especializado (pensamiento vigente históricamente en la enfermería). La humanización de los cuidados implica la mejora y perfeccionamiento de todo tipo de técnicas y aplicaciones de la tecnología que contribuyan al bienestar de la persona en el contexto de los cuidados, pero no se limita a esa dimensión, sino que la supera en aras al uso de la comunicación como herramienta de comprensión de significados del paciente y a la potenciación de su participación activa (en la línea de sus posibilidades) en sus procesos de salud-enfermedad. En definitiva esto implica la adopción de otra perspectiva (paradigma sociocrítico) en la que la enfermera no es una mera aplicadora de técnicas, sino que, además, actúa como agente de comunicación y comprensión de los significados que para el paciente tiene su proceso y, además, emplea la pedagogía y el pensamiento crítico para potenciar la implicación del paciente de forma activa y responsable.

\section{Educación superior, perfil profesional e investigación}

El rol de la educación superior en la construcción del perfil profesional de enfermería tradicionalmente se ha centrado en la técnica soslayando el carácter instrumental de la misma y obviando el sujeto esencial de la disciplina como núcleo central de investigación; es decir, el paradigma predominante en la enfermería 
en general y en la investigación de la disciplina en particular, ha sido el neopositivista (racional-tecnológico), en el que el profesional desempaña tareas de administración de técnicas de forma estandarizada mientras que el paciente se limita a un rol dependiente, pasivo $\mathrm{y}$ subordinado a la autoridad del técnico. Asimismo, la metodología predominante ha sido la experimental, cuantitativa $y$ estadística (Siles, 1999). Como es lógico, la investigación está influenciada por el perfil profesional neopositivista tanto en la investigación básica como en la aplicada. El enfoque positivista, que es el preponderante desde el origen de los métodos y usos científicos, ha constreñido la enfermería al ámbito del método experimental y la tecnología alejándola de su auténtico epicentro epistemológico.

\section{Investigación básica y aplicada en enfermería}

La generación de las teorías elaboradas con métodos experimentales que demuestran el funcionamiento de determinados fenómenos físicos constituye el núcleo principal de la investigación básica (Lozada, 2014). Estos procesos suelen transcurrir de forma lenta y duradera en el tiempo (requieren cierta perdurabilidad) para llegar a producir un tipo de conocimiento nomotético (generador de leyes). En consecuencia, las ideas vinculadas a la investigación experimental que permiten la producción de teorías es la predominante en la investigación básica, aunque en enfermería desde el punto de vista epistemológico -a pesar del estancamiento de la disciplina de los cuidados en el enfoque neopositivista- es subsidiaria y alejada de su núcleo central (el objeto-sujeto de la enfermería) (Siles, 1997, 2017; Amezcua, 2010).
Pero tampoco en la investigación aplicada -que es la encargada de buscar aplicaciones a las teorías que den respuestas a los problemas de la sociedad- la investigación en enfermería ha logrado traspasar los límites del neopositivismo y el tecnologicismo que siguen imperando en esta fase de investigación dedicada a la adaptación del conocimiento a las necesidades de la sociedad. En enfermería, la investigación aplicada, hasta ahora, se entiende de forma generalizada como estudios experimentales para demostrar la efectividad de tal o cual brebaje, emplasto, vendaje o utensilios en tal o cual tratamiento o problema. Algunos de estos estudios son autóctonos de la disciplina (Gallar et al., 2001), pero la mayoría son multidisciplinares (totalmente dependientes de la dirección de otros profesionales) ${ }^{1}$.

Planteada esta realidad, la cuestión es: ¿Los resultados derivados de estudios experimentales constituyen el producto principal de la investigación básica en enfermería?

\section{La humanización de los cuidados: El cuidado centrado en la persona como producto de investigación básica en enfermería}

Si limitamos la investigación aplicada o traslacional 2 a la producción de patentes y marcas y a productos con garantía de reversión económica en el contexto farmacéutico o médico industrial, esteremos haciendo un flaco favor a disciplinas tan imprescindibles como la enfermería. Efectivamente, si la investigación aplicada/traslacional supone la adaptación del conocimiento científico derivado de la investigación básica como baluarte científico para dar respuesta a las necesidades reales de la sociedad, ¿qué más precisan las enfermeras para identificar la 
deshumanización/despersonalización de los cuidados como un problema de gran calado que afecta a la sociedad de forma creciente? Tal como decía Protágoras en el siglo IV a. C.: El hombre es la medida de todas las cosas "Homo mesura", pero es el mismo hombre el que se empeña en ignorar esta tajante realidad (lo cual tampoco debe sorprendernos) (Figura I). Esto llevado al terreno de la enfermería no es sino centrar los cuidados en la persona (Siles, 2011). ¿Puede ser un producto de investigación aplicada el cuidado centrado en la persona?, ¿Es el cuidado centrado en la persona un producto necesario para aumentar el bienestar de la sociedad y contribuir a un aumento de su calidad de vida?, ¿qué beneficios sociales y económicos se pueden derivar de este producto en una sociedad cada vez más necesitada de cuidados?

\section{Incidencia de la síntesis transdisciplinar: Historia- Antropología-Enfermería en el uso de nuevos métodos $y$ enfoques: las narrativas como herramienta de humanización de los cuidados}

Antes de ponernos a emplear nuevos enfoques o métodos que den voz a los pacientes y potencien su participación, es necesario concienciarnos de una cuestión previa: el cambio de paradigma. Es preciso adaptar los principios del paradigma sociocrítico de Habermas (Siles, 1999, 2016a). Pero para que nuestro cambio trascienda las estrecheces de lo realizado por la moda en las nuevas tendencias académicas y sea, por el contrario, la consecuencia de una toma de conciencia y sensibilización con respecto a las necesidades reales que se perciben desde una perspectiva diferente a la neopositivista, debemos clarificar $y$ entender las características esenciales del paradigma sociocrítico que suponen la adopción de teorías y metodologías cualitativas donde la subjetividad adquiere un valor totalmente ignorado/despreciado en otros lindes científicos. Se trata de un paradigma que potencia la participación y emancipación de los sujetos investigados. Esto implica considerar al "paternalismo ilustrado": todo para el paciente pero sin el paciente; es decir, guardar la distancia, la objetividad y la diferencia de estatus de forma vertical donde el profesional es soberano y con la autoridad que le confiere su titulación, mientras el paciente no es más que un consumidor lego y sin nada que hacer en sus procesos de salud-enfermedad más que acatar de forma pasiva todas las prescripciones y técnicas dictaminadas por los profesionales.

Esto supone un giro de 180 grados en la mirada enfermera y el mismo ha sido posible, entre otras aportaciones, a la síntesis transdisciplinar (historia, antropología y enfermería) experimentada por la historia cultural de la enfermería. La historia es la primera disciplina que amplía sus métodos, teorías y enfoques con el fin de dejar de ser esclava de los datos concretos (árboles) y potenciar miradas sintéticas que perciban la globalidad del bosque estableciendo relaciones dinámicas entre diferentes factores que contribuyen al estado cambiante del bosque. La síntesis transdisciplinar de la que es tributaria la historia cultural de los cuidados se produce gracias al cruce de tres grandes escuelas: Frankfurt, Anales y Birminghan (Siles, 2018).

Las fuentes narrativas son almacenes de hechos (comportamientos, estilos de vida) y raíces de los hechos (creencias, valores, símbolos, significados y sentimientos) que constituyen el "por qué" de los hechos. Estas narrativas han sido usadas para extraer los 
testimonios y sus significados, siendo especialmente relevantes las narrativas autobiográficas escritas por personas que han vivido bajo las circunstancias marcadas por diferentes enfermedades: grandes quemados (Allué, 1996, 1997), cáncer (Zorn, 1991), sida (Gilbert, 1993), linfoma (Comas, 2009), insuficiencia renal y diálisis (Gracia Armendáriz, 2010), Sampedro, 1996).

\section{La enfermería basada en la narrativa y la poesía de los cuidados como producto de la investigación aplicada y complemento de la enfermería basada en la evidencia}

La EBN tiene en cuenta los procesos de construcción social de la experiencia que se derivan de las vivencias y puede servir de puente entre la objetividad científica de la EBE (Siles, 2005, 2018; Siles y Solano, 2014; Amezcua, 2018). Para un prostático ante la perspectiva de la palpación el diagnóstico es HBP pero él se muere de vergüenza (sentimiento-vivencia); para un paciente cuyo diagnóstico es sida, la discriminación, miedo e incertidumbre constituyen sentimientos que indicen de plano en el proceso de diagnóstico o tratamiento. En cualquier caso, en la EBN el profesional debe partir de la contemplación de una realidad tan compleja como la persona ${ }^{3}$ articular la comunicación como herramienta de comprensión de significados que tiene el proceso de salud enfermedad para el paciente y buscar los mecanismos precisos para, en la medida de sus posibilidades y circunstancias, implicarlo activamente de forma que su participación resulte crucial4.

En definitiva, tal como afirma Ricoeur (1995) ante la amenaza del "no sentido" que provocan los cambios que afectan la homeostasis fisiológica, psicológica o social de las personas, las tramas narrativas constituyen el medio por el cual reconfiguramos nuestra experiencia para llegar a comprendernos en diferentes situaciones.

David Sacket, uno de los padres de la MBE 5 ya a mediados de los noventa alertó sobre las consecuencias negativas del uso generalizado de la misma alegando que debía restringirse a especialistas preparados y con el tiempo suficiente de manera; es decir que no formara parte de la práctica generalizada de los médicos clínicos para no sobrecargarlos aún más y que no repercutiera negativamente en la relación médico paciente. En la órbita de la medicina surgieron iniciativas para el desarrollo de la MBN (Kleinman, 1989; Greenhalgh, 1999), y paralelamente se fue desarrollando la EBN느.

La poesía de los cuidados constituye otra herramienta complementaria a la EBN en la medida que facilita la contemplación de los sentimientos que se producen durante la interacción paciente-enfermera. Consiste en la expresión de los sentimientos que emergen durante la interacción pacienteenfermera. No hay que obviar el hecho demostrado de que una cuestión estética, como los sentimientos constituyen la principal fuente de motivación en la orientación de conductas y en la generación de conocimientos y actitudes, con todo lo que ello conlleva en educación para la salud (Siles, et al., 2016b). Siles y Solano (2016b) analizan el arte de enfermería desde la perspectiva estética y describen la materia prima compleja, delicada y única que es simultáneamente objeto-sujeto (homo dúplex) de enfermería: el ser humano. De forma que la obra artística de la enfermería consiste en la aplicación del cuidado a la frágil y sensible materia prima pudiendo llegar a experimentar momentos bellos o sublimes. No hay arte más privilegiado dado el valor de su materia prima. Dicho de 
otra forma, la más importante aportación de la poesía a la enfermería radica en la clarificación de su naturaleza transcendente mediante la revelación de su objeto sujeto: el ser humano. La persona como núcleo, como materia prima con la que trabaja la enfermera para "producir su obra" (Siles \& Solano, 2016b).

Diversos autores han descrito la utilidad de la poesía en enfermería: Para Watson (2008) la poesía de los cuidados es la que permite encontrar la verdad trascendente del acto enfermero; Homes \& Gregory (1998) sostienen que es una fuente de conocimiento para enfermería; Birx (1994) señala que la poesía es un instrumento para analizar la trascendencia de encuentros/interacciones especialmente intensos entre las personas. Es cierto que el carácter subjetivo de la expresión de los sentimientos supone una barrera para el encuentro con la ciencia, pero para vadear ese obstáculo que parecía insalvable ha surgido la sociopoética que permite el estudio de la expresión de los sentimientos de forma intersubjetiva alcanzando de esa forma cierta objetividad (Siles, 2014). La sociopoética ha sido aplicada en la producción de conocimiento enfermero por diversos autores: en estudios con adolescentes (Paiva Moraes et al., 2003), varios trabajos que sirven como puente entre la práctica enfermera y la investigación (Santos, 2005), o en la investigación de la gestión de emociones y sentimientos con alumnos de enfermería (Siles y Solano, 2017).

\section{BIBLIOGRAFÍA}

- Allport, G. (1985). Personalidad, su configuración y desarrollo. Barcelona: Herder.

- Allport, G. (1985). Personalidad, su configuración y desarrollo. Barcelona: Herder.

- Allué, M. (1996). Perder la piel. Barcelona: Seix Barral.
- Allué, M. (1997). «Sobrevivir en la UCI». Enfermería Intensiva, 6, 29-34.

- Amezcua, M. (2010). «Investigación Aplicada en Cuidados de Salud». Index de Enfermería, 19(4), 237$239 . \quad$ Recuperado de http://scielo.isciii.es/scielo.php?script=sci_arttext\& pid=S1132-12962010000300001\&lng=es\&tlng=es.

- Amezcua, M. (2018). En primera persona. Narrativas de pacientes con ostomía. Granada. Fundación Índex.

- Birx, E. (1994). The poetry of nursing. Clin Nurse Spec 8, 292-293.

- Comas, J. (2009). Crónicas del linfoma. Madrid: Rey Lear.

- Gallart, E. et al. (2001). Estudio experimental para comprobar la efectividad de los ácidos grasos hiperoxigenados en la prevención de las úlceras por presión en pacientes ingresados. Enfermería Clínica, 11(5), 179-238.

- Gilbert, H. (1992). El protocolo compasivo. Barcelona: Tusquets.

- Greenhalgh, T. (1999). Narrative based medicine: Narrative based medicine in an evidence based world. BMJ Clinical Research, 318(7179), 323-325 DOI:10.1136/bmj.318.7179.323)

- Holmes, V., Gregory, D. (1998). Writing poetry: a way of knowing nursing. J Adv Nurs 8(6):1191-4.

- Kleinman, A. (1989). The Illness Narratives: Suffering, Healing, And The Human Condition. New York: Basic Books.

- Lozada, J. (2014) Investigación Aplicada: Definición, Propiedad Intelectual e Industria. Ciencia América, 3, 34-39.

- Paiva Moraes, L. M., Batista Braga, V. A., Carneiro Sileveria, L. (2003) Socipoética e enfermagem: una aproximación en la investigación con adolescentes. Revista Baiana da Enfermagem. 18: 89-96.

- Ricoeur, P. (1995). Sí mismo como otro. Madrid: Siglo XXI.

- Sackett, D., Rosenberg, W. MC., Muir Gray, J. A., Haynes, R .B. \& Richardson, W. S. (1996). Evidence based medicine: what it is and what it isn't. BMJ, 312(71).

DOI: https://doi.org/10.1136/bmj.312.7023.71 (Published 13 January 1996).

- Sampedro, R. (1996). Cartas desde el infierno. Barcelona: Planeta.

- Santos, I. (2005) Sociopoética: un puente para "cuidar -investigar" en enfermería. Index Enferm 14 (50): 35-37. http://dx.doi.org/10.4321/S1132$\underline{12962005000200007 .}$.

- Siles, J. \& Solano, C. (2014). Qualitative Research and Narrative Sources in the Context of Critical and Renal Cares. Open Journal of Nursing, 4(7), 385-401 DOI: 10.4236/ojn.2014.46045.

- Siles, J. \& Solano, C. (2016b). Sublimity and beauty: 
A view from nursing aesthetics. Nursing Ethics, 23(2), 154-166.

- Siles, J. (1999). Epistemología y enfermería: Por una fundamentación científica y profesional de la disciplina. Enfermería Clínica, 7(3): 38-44.

- Siles, J. (2011). Historia de la enfermería. Madrid: DAE.

- Siles, J. (2014). El humanismo en enfermería a través de la sociopoética y la antropoética. Cultura de los Cuidados (Edición digital) 18(38), 9-13. doi.org/10.7184/cuid.2014.38.01.

- Siles, J. (2016a). La utilidad práctica de la Epistemología en la clarificación de la pertinencia teórica y metodológica en la disciplina enfermera. Index de Enfermería, 25(1-2). Recuperado de http://www.indexf.com/index-enfermeria/v25n12/251215.php.

- Siles, J. (2018). Educación Superior e Investigación Aplicada: sinergias y conveniencias [video]. Rev Paraninfo Digital, 28. Recuperado de http://www.index-f.com/para/n28/v005.php.

- Siles, J. et al. (2005). Narrative anthropology and some health-life situations in Benedetti and Octavio Paz`s works. Cultura de los cuidados. 17(9), 59-67.

- Siles, J. \& Solano, C. (2017). Poesía y cuidados: un instrumento para la gestión de emociones y sentimientos en enfermería. Enfermería: Cuidados Humanizados, 62 6 2393-6606. doi.org/10.22235/ech.v6i2.1463.

- Watson (1996) afirma que la poesía es una vía pertinente para identificar la verdad.

- Watson, J. (2008). Nursing: The Philosophy and Science of Caring. Colorado: University Press of Colorado.

- Zerhoni, E.A. (2005). Translational and clinical science-time for a new vision. J Med 353, 1621-324.

- Zorn, F. (1991). Bajo el signo de Marte. Barcelona: Anagrama.

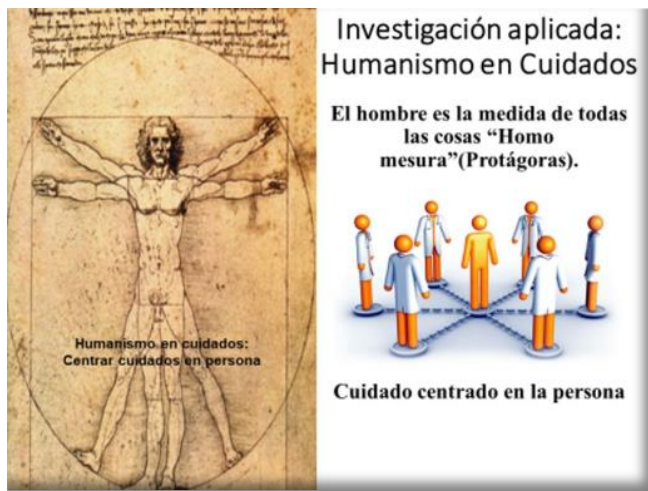

\section{NOTAS}

(1) La multidisciplinaridad en ciencias de la salud es el nivel de colaboración entre distintos tipos de profesionales/científicos, en el que la jerarquización del trabajo parte de una dirección (generalmente médica) que apenas hace partícipes a los profesionales de enfermería de los pormenores de la investigación. Éstos apenas tienen la información estrictamente necesaria para realizar sus tareas puramente técnicas (extracciones de muestras, aplicaciones de medicamentos, etc.). De este nivel de jerarquización estrictamente vertical a la transdisciplinaridad, donde se produce una síntesis que es el producto del intercambio de conocimientos e informaciones entre profesionales de diferentes disciplinas en un nivel de igualdad jerárquica similar a la horizontalidad.

(2) La necesidad de superar los puentes entre las dos orillas de la investigación, la básica y la clínica, dio lugar a la investigación traslacional. Para algunos autores este es un neologismo y barbarismo innecesario dado que el concepto "investigación aplicada" puede ser, en esencia, lo mismo, pero para otros autores, la investigación traslacional se ciñe mucho más a los problemas clínicos que la aplicada (más relacionada con las industrias farmacéutica y médica) (Zerhoni, 2005).

(3) Para Gordon Allport, la Individualidad es la característica esencial de la naturaleza humana, mientras que la personalidad constituye la organización dinámica de los sistemas psicofísicos que determina una forma particular de pensar y actuar en su proceso de adaptación al medio (Gordon Allport 1985).

(4) Los programas marco de la Comunidad Europea contemplan en sus líneas prioritarias la necesidad de la participación del paciente: En Ciencia con y para la sociedad: "Es necesaria una cooperación efectiva entre ciencia y sociedad para poder sumar nuevos talentos a las filas de la ciencia y emparejar la excelencia en este campo con la conciencia social y la responsabilidad" (Horizonte 2020, 17).

(5) Medicina Basada en la Evidencia.

(6) Después de mucho analizar la función de las narrativas en el contexto de la salud y reflexionar sobre las definiciones aportadas por diversos autores, mi interpretación sobre la Enfermería Basada en la Narrativa es la siguiente: "La EBN consiste, por un lado, en la consideración de la subjetividad del paciente como evidencia integrada en sus narrativas sobre procesos de salud-enfermedad $\mathrm{y}$, por otro, en la comprensión y análisis de los significados subyacentes a las mismas contribuyendo a la humanización de los cuidados". 